\title{
Nucleotide Biochemistry
}

National Cancer Institute

\section{Source}

National Cancer Institute. Nucleotide Biochemistry. NCI Thesaurus. Code C19400.

The branch of biochemistry that is specifically concerned with the structure, synthesis,

maintenance and biological pathways that involve nucleotides. 